\title{
Étude en Rusitec de l'influence du pH sur la synthèse ruminale de thiamine
}

\author{
L Alves de Oliveira, A Durix, S Bony, C Jean-Blain
}

INRA-ENVL, unité Physiopathologie du rumen, 1, avenue Bourgelat, BP 83, 69280 Marcy-L'Étoile, France

L'effet des régimes acidogènes, riches en glucides fermentescibles, sur la synthèse microbienne de thiamine dans le rumen a été très peu étudié.

Nous avons utilisé un fermenteur semi-continu de type Rusitec ensemencé avec un inoculum provenant de 3 moutons nourris avec $1 \mathrm{~kg} \mathrm{de}$ foin, $100 \mathrm{~g}$ d'orge et $10 \mathrm{~g}$ d'urée. Des conditions de $\mathrm{pH}$ bas ont été obtenues par l'apport journalier de $17 \mathrm{~g}$ MS d'un aliment purifié exempt de thiamine séjournant $48 \mathrm{~h}$ dans le fermenteur $(4 \mathrm{~g}$ de cellulose pure, $4 \mathrm{~g}$ d'amidon de blé, $4 \mathrm{~g}$ d'amidon soluble, $2,4 \mathrm{~g}$ de pectines, $1,6 \mathrm{~g}$ de xylane, $1 \mathrm{~g}$ d'urée). L'un des fermenteurs reçoit une salive artificielle de Mac Dougall normale (SN), l'autre une salive modifiée (SR) dans laquelle les quantités de bicarbonate et de phosphate sont réduites respectivement de $25 \%$ et $41 \%$ par rapport à $\mathrm{SN}$. L'évolution du $\mathrm{pH}$ est enregistrée sur $24 \mathrm{~h}$ à la sortie des fermenteurs. Après une semaine d'adaptation, on mesure quotidiennement la production d'acides gras à courte chaîne (AGCC) et la composition des gaz. La protéosynthèse microbienne et la thiamine (dosée par fluorimétrie) sont estimées dans les effluents et les résidus des sacs.

L'utilisation de SR par rapport à SN provoque un abaissement de $\mathrm{pH}$ de 0,5 unité, une diminution de la production des AGCC et une modification de leurs proportions molaires (tableau I) sans toutefois que l'on détecte d'acide lactique. Environ $6 \mathrm{~h}$ après l'introduction du substrat, le $\mathrm{pH}$ dans les fermenteurs atteint un minimum de 5,8 et 5,6 respectivement pour SN et SR sans avoir d'incidence sur la production journalière de méthane. Contrairement aux résultats obtenus au cours d'incubations de $6 \mathrm{~h}$ par Bick et al (1978), nous observons une synthèse nette de thiamine avec ce modèle. Nous avons mis en évidence que toute la thiamine est localisée dans les bactéries. La production plus élevée de thiamine au $\mathrm{pH}$ le plus bas, significative même si on la rapporte à l'azote incorporé par les microbes (Ni), peut s'expliquer par une efficacité accrue de la synthèse de thiamine dans les bactéries ou par une sélection, par le $\mathrm{pH}$, de bactéries plus aptes à synthétiser cette vitamine.

Bick S, Breves G, Höller H (1978) Zeits Tierphysz Tierernähr Futtermitt 41, 8-17

Tableau I. Effet du pH sur l'activité fermentaire et la production de thiamine (moyenne \pm erreur standard, $n=9$ ).

\begin{tabular}{|c|c|c|}
\hline Salive & $S N$ & $S R$ \\
\hline pH effluents & $6,62 \pm 0,05^{a}$ & $6,13 \pm 0,14^{b}$ \\
\hline AGCC (mmoles/j) & $125,1 \pm 2,7^{a}$ & $110,1 \pm 2,6^{b}$ \\
\hline cétate $(\%)$ & $52,3 \pm 1,5^{a}$ & $63,7 \pm 1,5^{b}$ \\
\hline opionate (\%) & $39,0 \pm 2,0^{a}$ & $21,0 \pm 2,3^{b}$ \\
\hline Butyrate (\%) & $3,9=$ & $6,4 \pm$ \\
\hline Valérate $(\%)$ & $1,5 \pm 0$ & $2,3 \pm 0,14^{b}$ \\
\hline Isovalérate (\%) & $1,1 \pm 0,07^{a}$ & $2,8 \pm 0,20^{b}$ \\
\hline Caproate (\%) & $0,031 \pm 0,02^{a}$ & $0,9 \pm 0,2^{b}$ \\
\hline $\mathrm{CH}_{4}$ (mmoles $\left./ \mathrm{j}\right)$ & $25,3 \pm 1,8^{a}$ & $21,8 \pm 2,3^{a}$ \\
\hline & & $285 \pm 10,3^{a}$ \\
\hline Thiamine ( & $100,2 \pm 4,4^{a}$ & $129,0 \pm 4,6^{b}$ \\
\hline Thiamine/Ni ( $\mu \mathrm{g} / \mathrm{mg})$ & $0,32 \pm 0,02^{a}$ & $0,46 \pm 0,03^{b}$ \\
\hline
\end{tabular}

Dans une même ligne, ${ }^{a} \neq b(p \leq 0,05 ;$ test $t$ de Student $)$. 